\title{
PRESAGING CARDIAC PAIN
}

\author{
BY \\ WILLIAM EVANS \\ From the Cardiac Department of the London Hospital, London E.1
}

Received March 12, 1965

When the electrocardiogram was recorded in 658 consecutive symptom-free men of 35 years of age or older, $108(16 \%)$ showed abnormalities of the tracing consistent with the presence of cardiac infarction, limited at the time to a small sector of the myocardium (Evans, 1961). These men were not recalled for further examination as it was felt that it might give rise to a degree of unwarranted invalidism in many of them. Up to that time, no patient, the subject of cardiac pain from cardiac infarction, had been seen in whom a cardiogram recorded fortuitously during three years preceding the onset of pain had been a normal tracing. These findings seemed to justify the conclusion that the cardiogram can presage cardiac pain arising from occlusion of a coronary artery.

\section{THE INVESTIGATION}

The present paper describes an inquiry which contrived to test the validity of this deduction. It concerns 38 patients who attended for examination on two separate occasions. There were 30 men and 8 women whose ages varied from 42 to 78 years, mean age 59 . The mean age of the 8 women was 61 years.

The reasons for their first attendance were diverse and included immaterial palpitation, tiredness, dizziness, and faintness, but many came for what they termed a routine "check-up". None attended on account of symptoms having a cardiac source, while special care was taken to exclude from the series any who complained of chest pain, even though it might have presented atypical features, lest it should have arisen from coronary arterial disease.

Except in 3 patients with systemic hypertension, both clinical and radiological examinations found no abnormal signs.

The electrocardiogram consisted of leads I, II, III, IIIR, CR1, CR4, and CR7; if the R wave was absent in CR1, CR2 was added. In each patient the electrocardiogram was abnormal, but as none of them had cardiac pain, they received reassurance and were not invited to attend for re-examination. It was ascertained that no patient was taking digitalis at the time. When these 38 patients did attend subsequently because cardiac pain had set in, another electrocardiogram was recorded and was compared with the previous tracing.

At this second examination the nature of the chest pain appeared to typify cardiac pain in that it was in the region of the sternum sometimes extending to other areas, it was non-lancinating in character, and was induced by physical exercise, especially walking quickly or over rising ground. Not infrequently it occurred at rest as well. Exceptionally, such cardiac pain had been present for some time before the patient attended for the second examination and in 6 the period had been two years or more, but usually the patient had sought an explanation of his symptom much earlier, for in the remaining 32 the average intervening period had been 3 months. 


\section{Premonitory Pre-Pain Electrocardiogram}

The electrocardiograms recorded at the patients' first attendance were consistent in one respect, namely that in the 35 uncomplicated cases the tracing never exhibited the gross deformities identified with the salient kind of cardiac infarction where the $T$ waves might be deeply inverted and the $\mathrm{Q}$ waves prominent. Instead the changes were those which have been described as the lesser deformities that result from the more limited kind of cardiac infarction. In 3 patients inversion of the $T$ wave in leads CR7 and I stood for left ventricular preponderance associated with systemic hypertension; in 2 of these, changes indicative of added infarction were again minor ones (Fig. 1), but there was frank inversion of the T wave in CR4 in the initial cardiogram in the third instance.

It was expected that the design of the electrocardiogram obtained at the first examination before cardiac pain had set in would not be different from that in those instances where pain was present when the infarct continued to be limited in its distribution, and the changes are conveniently considered here in relation to the 7 consecutive segments of the single-beat electrocardiogram.

$Q$ Wave. In one a $\mathrm{Q}$ wave appearing in extrasystoles was the chief clue. In another a $\mathrm{Q}$ wave was present in CR1 and CR2 from a septal lesion, but this abnormality was accompanied by small changes elsewhere in the tracing. There was no example in this series of a $Q$ wave, absent in III, appearing in IIIR.

RS Limb. A notch on the lower part of the RS stem in CR7 was present in only one patient, and in this instance it was accompanied by depression of the S-T segment in IIIR as additional evidence of limited cardiac infarction. This notching of the RS is an exaggerated form of slurring which is part of the pattern belonging to the trough variety of depression of the S-T segment (Fig.2).

$S$ Wave in Leads II and III. A deep S wave in leads II, III, and IIIR, in the absence of an S wave in lead I, was a lone sign of antero-lateral cardiac infarction in 2 patients (Fig. 3), and in another 2 the abnormality was accompanied by S-T depression in other leads.

$S-T$ Segment. Depression of the S-T segment was the most common abnormality, being present in 26 patients. It was confined to a single lead, either IIIR or CR4, in 3 instances; it occurred in 2 leads in 1 patient, 3 leads in 1, 4 leads in 7, 5 leads in 4 when CR1 and CR4 were the unaffected leads, and in 6 leads in 10 patients where CR1 was the exempted lead.

The depression was one of two kinds, either a trough or plane variety, and though the former was less common, it is of equal importance on account of the ease with which it may go unrecognized.

Trough or Sickle Depression. In this kind the deformity involves the early part of the S-T segment. Should the $\mathbf{S}$ wave be absent, the terminal portion of the RS stem is slurred as it slants to emerge into a concave $\mathbf{S}-\mathrm{T}$ sector. When the $\mathrm{S}$ wave is present, the trace dips after the returning limb of the $\mathrm{S}$ reaches the isoelectric level. In either event the $\mathrm{S}-\mathrm{T}$, as it continues into the ascending limb of the $T$ wave, forms a curve or trough resembling a fishhook or a sickle. The base of the depression need not, and usually does not, reach below the isoelectric line, and may even be raised above it, while the $T$ wave beyond is often of natural height; because of this it may hinder a recognition of the deformity (Fig. 4 and 5).

Plane S-T Depression. In this instance the deformity involves the terminal portion of the S-T segment. As its name suggests, the trace from its commencement at the $S$ wave does not deviate from the horizontal plane for a distance of $0.1 \mathrm{sec}$. or over until it reaches. the ascending limb of the $T$ wave. Here also the segment need not be depressed below the isoelectric level, while the $T$ wave beyond may be of natural or even large amplitude (Fig. 6).

$T$ Wave. A low and blunt T wave in leads I and CR4 or CR7 was the only cardiographic deformity in 3 patients, when a positive exercise test confirmed the significance of the change. Terminal dipping of the $T$ wave as a premonitory electrocardiographic sign of cardiac pain was met with in one patient, but not as a lone deformity. 

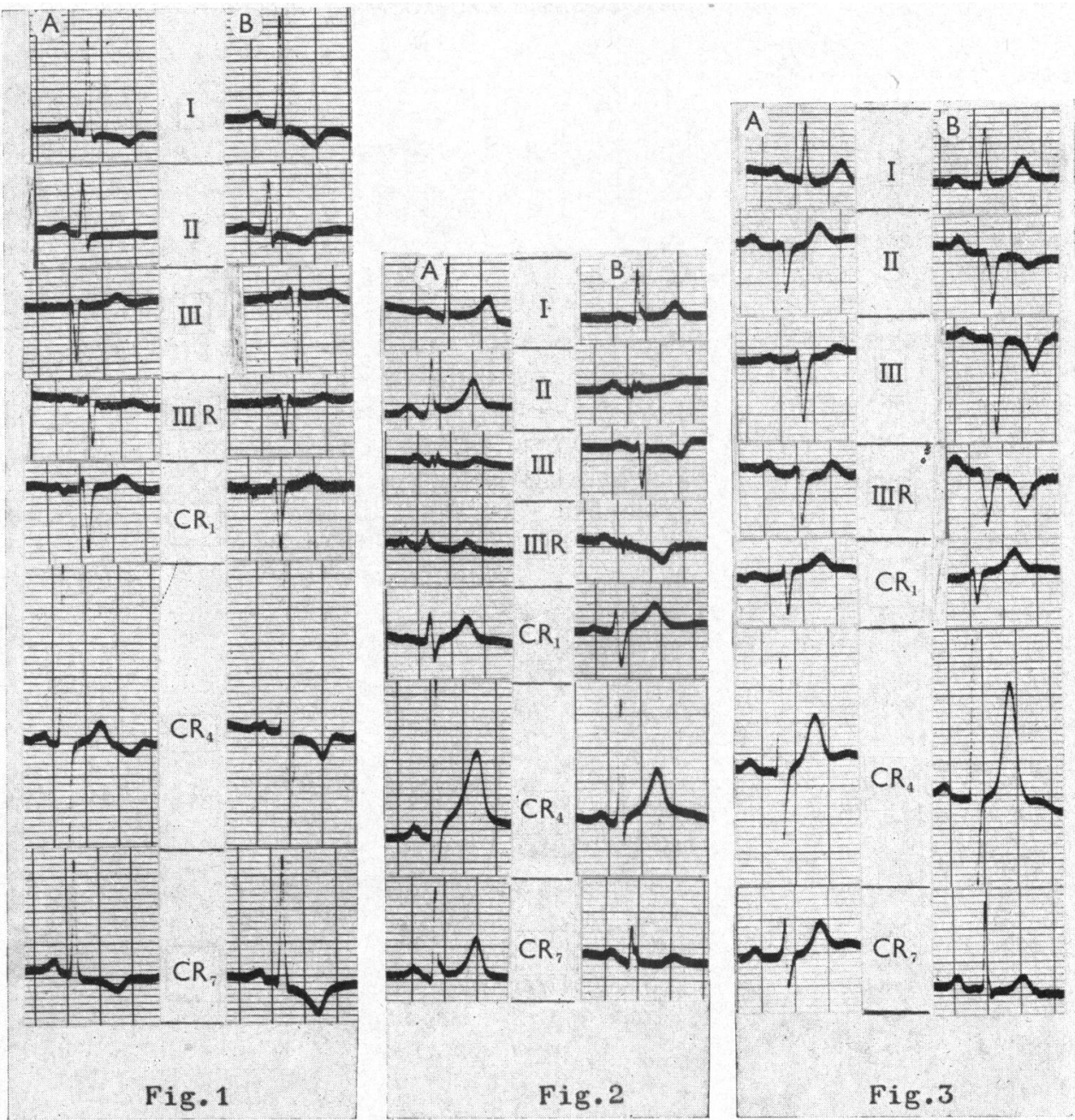

FIG. 1.-In (A), recorded 18 months before the onset of cardiac pain, the S-T segment is depressed in leads CR7 and I, and the U wave in CR4 is inverted; these signs were accepted as evidence of left ventricular hypertrophy from systemic hypertension. Associated deep inversion of the T wave in lead I, along with S-T depression in IIIR, suggested the addition of cardiac infarction. In (B), recorded a month after the advent of cardiac pain, inversion of the $T$ wave has been added in leads II and CR4. From a man aged 50 years.

FIG. 2.-In (A), recorded 3 years before the onset of cardiac pain, there is a trough depression of the S-T segment in leads II, III, IIIR, and CR7. In (B), recorded 3 years after the advent of cardiac pain, the $T$ wave is depressed or inverted in these leads, and a notch has appeared on the RS stem in station 7. From a man aged 52 years.

FIG. 3.-In (A), recorded one year before the onset of cardiac pain, the $S$ wave is deep in leads II, III, and IIIR and absent in I. In (B), recorded 15 days after the advent of cardiac pain, deep Q waves and inverted $T$ waves have appeared in these leads, while the $T$ has become depressed in CR7. From a man aged 76 years.

$T-U$ Segment. Depression of the $\mathrm{T}-\mathrm{U}$ segment was encountered once, but not as a lone sign, for it was associated with S-T depression in several leads (Fig. 6).

$U$ Wave. Inversion of the $U$ wave in the premonitory electrocardiogram in one patient was probably part of the pattern of left ventricular preponderance from systemic hypertension, rather than proof of coronary arterial disease (Fig. 1). 

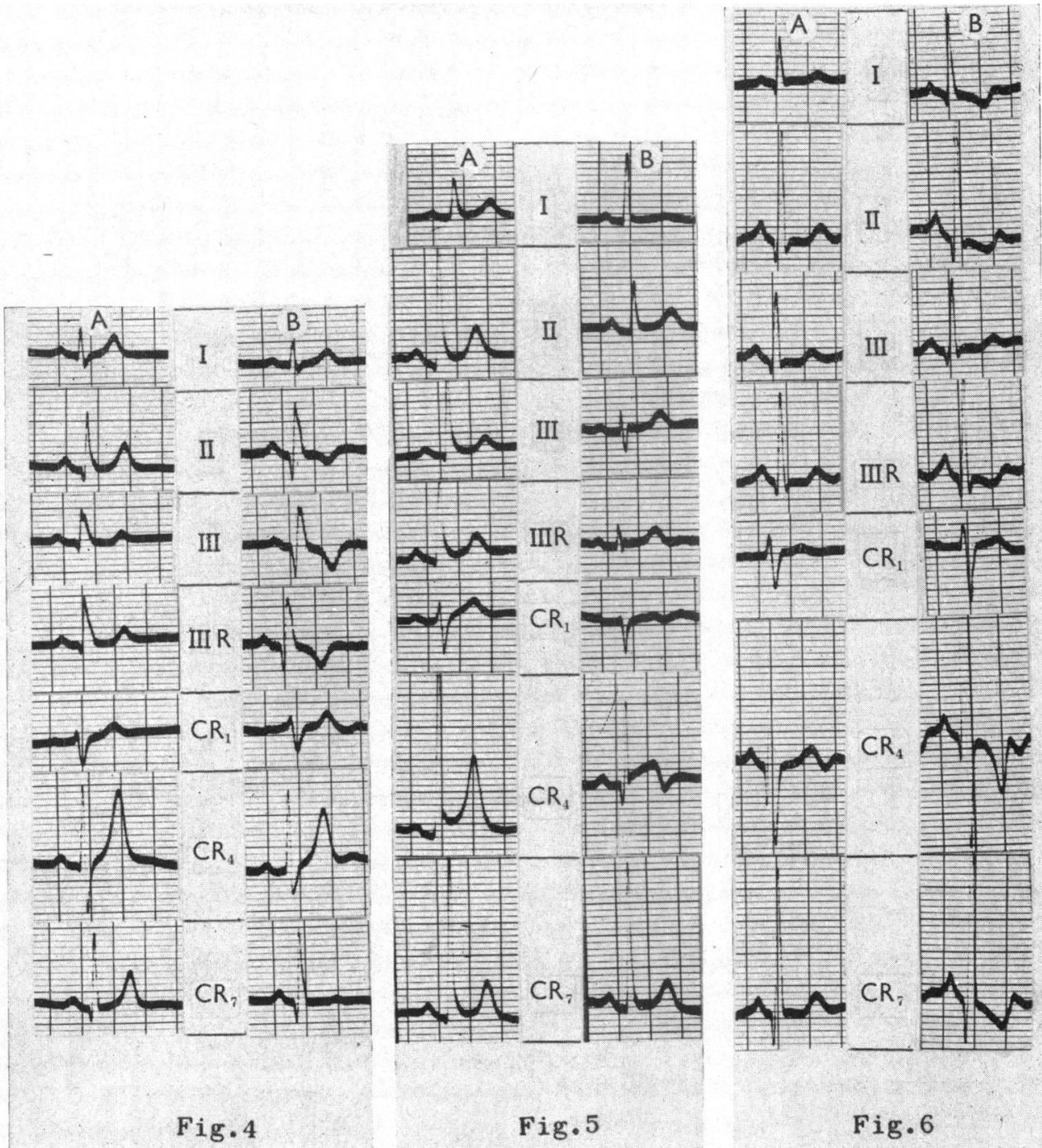

Fig. 4.- In (A), recorded 3 years before the onset of cardiac pain, there is trough depression of the S-T in lead II, and plane depression in leads III and IIIR. In (B), recorded 18 months after the advent of cardiac pain, deep $Q$ waves and inverted $T$ waves have appeared in these leads, while the $T$ wave is flat in CR7. From a man aged 58 years.

Fig. 5. - In (A), recorded 5 years before the onset of cardiac pain, there is trough depression of raised S-T segments in leads II, III, IIIR, CR4, and CR7. In (B), recorded one year after the advent of cardiac pain, there is plane depression of the S-T in leads II, III, and IIIR, a flat T wave in lead I, and terminal dipping of the $T$ wave with elevation of the $S-T$ segment in lead CR4. From a man aged 62 years.

FIG. 6. - In (A), recorded 4 years before the onset of cardiac pain, there is plane depression of the S-T segment in leads I, II, III, IIIR, and CR7, with depression of the T wave in leads I and CR4, and depression of the T-U segment in CR4. In (B), recorded 2 years after the advent of cardiac pain, there is deep inversion of the $T$ wave in several leads. From a man aged 51 years.

In one uncomplaining subject sent for a routine examination, the presence of a right bundlebranch block was regarded as an immaterial finding, but when he attended subsequently on account of chest pain the bundle lesion was considered to have resulted from coronary arterial disease, because other cardiographic signs had then been added as evidence of this. 


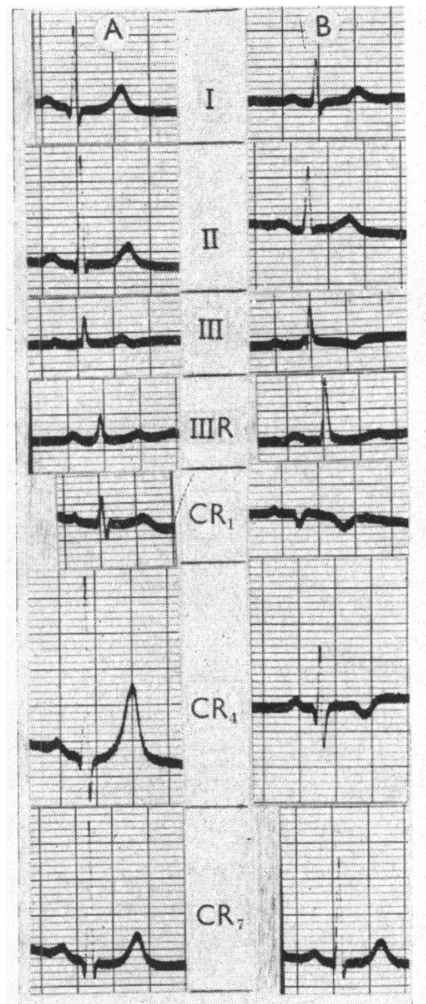

Fig. 7

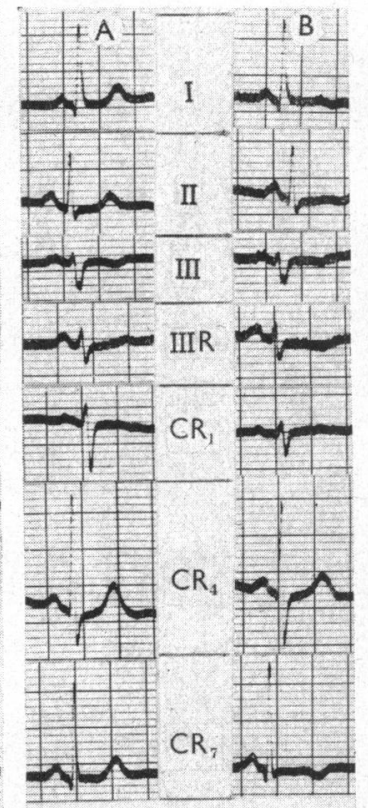

Fig. 8
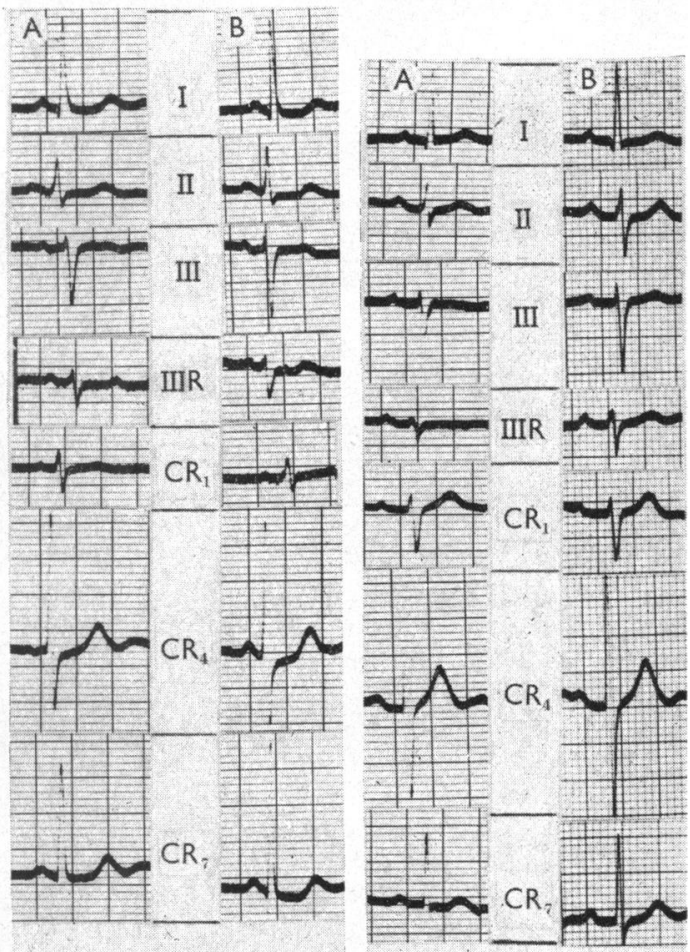

Fig.9

FIG. 7.-In (A), recorded 9 months before the onset of cardiac pain, there is plane depression of the $\mathrm{S}-\mathrm{T}$ in leads I, II, and CR7. In (B), recorded 5 years after the advent of cardiac pain, inversion of the $\mathrm{T}$ wave in leads III, CR1, and CR4, are additional deformities. From a man aged 48 years.

FIG. 8.- In (A), recorded 4 months before the onset of cardiac pain, there is plane S-T depression in leads I and CR7, and a degree of depression of the S-T in IIIR. In (B), recorded 6 months after the advent of cardiac pain, the $T$ wave is fiat in all the limb leads and is inverted in CR7. From a woman aged 54 years.

FIG. 9.-In (A), recorded 4 years before the onset of cardiac pain, there is plane S-T depression in leads I, II, IIIR, and CR7, with a low T wave in CR4. In (B), recorded 7 months after the advent of cardiac pain, the S-T depression is increased in CR4 and CR7. From a woman aged 69 years.

Fig. 10.- In (A), recorded 8 years before the onset of cardiac pain, the $T$ wave is low and blunt in leads I, II, and CR7. In (B), recorded 2 weeks after the advent of cardiac pain, pathologically deep S waves have appeared in leads II and III. From a man aged 53 years.

\section{Ultimate or Post-pain Electrocardiogram}

In only 2 patients had the premonitory electrocardiogram, which showed S-T depression, remained stationary after the advent of pain. Among the remaining 36 patients, the $T$ wave had become inverted in 18 (Fig. 7 and 8), the S-T segment showed greater depression in 12 (Fig. 9), and in 6 patients the added changes consisted of right bundle-branch block in 2, a deep S wave in leads II, III, and IIIR in 2 (Fig. 10), atrial fibrillation in 1, and in another case a $Q$ wave had appeared in leads CR1 and CR2.

In 4 instances, the newer changes indicated a contrecoup effect from added infarction in an opposite sector of the left ventricle, while in the remaining patients they stood for a localized extension of the lesion within the sector, or its spread to an adjoining sector. 
The time interval between finding a specific abnormality in the pre-pain eletcrocardiogram and the onset of cardiac pain was a year or more in 35 of the 38 cases. The interval exceeded 2 years in 32 , 3 years in 7,10 years in 5,13 years in 3, and 14 years in one patient. Naturally, these time intervals are minimal values for they do not take into account the period during which graphically discoverable coronary arterial disease had existed before the first electrocardiogram was recorded in each patient.

These findings appear to establish that the abnormal electrocardiogram is always an antecedent of cardiac pain. In that the investigation was designed to include only those patients who eventually developed cardiac pain, it cannot be affirmed that everyone with this abnormal cardiogram is destined eventually to contract cardiac pain from extension of the limited infarction which is then present, but it does show that the complication threatens even after years of apparent immunity from it, so that it was 6 years before it set in in 13 patients, and as long as 10 years in 5 patients. Among such patients in whom so many years had intervened between the initial casual electrocardiogram and the onset of cardiac pain, it was expected that a few at least would show a normal tracing. The fact that none did is regarded as highly significant.

The exercise electrocardiogram, should it show exaggerated changes, will select those patients in whom the onset of pain from extension of infarction is likely to be imminent, but in the absence of such prominent changes the test cannot then be regarded as indicating that this complication is to be long postponed. There is need for research into this limited problem, for it has already been shown that the degree of S-T depression resulting from exercise does contribute to an estimate of the prognosis. Thus, Robb and Marks (1964) have stated that a slight degree of depression (under $1 \mathrm{~mm}$.) has twice, moderate depression five times, and a depression greater than $2 \mathrm{~mm}$. twenty times, the expected standard mortality. Personal experience with the exercise cardiogram has also suggested that in patients showing the lesser cardiographic signs of limited cardiac infarction, the difference between those with and those without cardiac pain rests with the efficiency of the remaining and collateral coronary circulation.

\section{Discussion}

In that it can now be affirmed that the electrocardiogram can detect coronary arterial disease unerringly in its presymptomatic phase, there is need to examine the application of this newer knowledge in practice.

In the first place it imposes a discipline to be familiar with the lesser electrocardiographic signs which denote a limited injury to the myocardium, which follows its diminished blood supply because of coronary arterial disease. Through electrocardiography this pathological state yields to diagnosis earlier than the appearance of cardiac pain. In these days when the routine examination of symptomless men in industry and commerce is failing to uncover lethal coronary arterial disease during life (Schor et al., 1964), there is need to emphasize that the clinical recognition of this lurking threat will be expedited not through the introduction of more electrocardiographic leads, but through the exercise of more care in reading the tracing recorded in a limited number of standard leads.

When the findings of this investigation are considered in relation to treatment, if it were possible for the march of coronary atherosclerosis to be halted by any therapeutic procedure, a place for routine electrocardiography will have been established, but until such time, the advantages belonging to the presymptomatic recognition of coronary arterial disease are negligible. Again, if the surgical treatment of coronary arterial disease is ever entertained as a practical measure, to uncover it before cardiac pain has set in would be an insurance that the myocardial injury is limited in its distribution, but the exact state of the coronary arterial tree, whether showing localized or more generalized narrowing, cannot be accurately told by the electrocardiogram.

In the case of an applicant for life insurance, or for entry into a superannuation scheme, or in one prior to acceptance into any occupation, should the electrocardiogram uncover symptomless coronary arterial disease, it would be unethical for that reason to act presumptiously and disadvantageously 
to the patient, because in the meantime the prognosis belonging to the individual tracing is not known. Certainly, it is from among this group that cardiac pain from extension of cardiac infarction occurs, but since no set treatment has so far given unequivocal promise of halting the progress of coronary arterial disease, to inform an uncomplaining subject, in whom a fortuitous electrocardiogram discloses coronary disease, of the finding, is considered to be most undesirable. On the other hand, should the electrocardiogram prove to be a strictly normal tracing, the presence of critical coronary arterial disease is thereby excluded, and this reassurance should not be withheld from the patient, for the conveying of such good tidings is balm in the hands of the cardiologist with which to soothe and calm many an anxious patient.

\section{SUMMARY AND CONCLUSIONS}

In 38 patients with cardiac pain in whom the diagnosis of cardiac infarction had been confirmed by the electrocardiogram, it happened that a previous tracing had been recorded fortuitously, and often some years before the onset of cardiac pain.

When the first electrocardiogram was taken, the attendance was usually for a routine examination of a symptomless subject, sometimes in one with immaterial symptoms, but never in one with chest pain or material dysrhythmia. In each instance this initial electrocardiogram had shown the lesser changes identified with cardiac infarction which at the time was limited to a small sector of the myocardium. This information was not conveyed to the patients, and they were not invited to attend for further examination.

Each of these 38 patients, however, returned subsequently because cardiac pain had set in. The length of the intervening period varied. With three exceptions it was a year or more in every case, and it exceeded 2 years in 32 cases. In 13 the onset of cardiac pain had been delayed for 6 years, and for over 10 years in 5 patients.

The ultimate electrocardiogram, recorded at the second examination following the advent of cardiac pain, had remained stationary in only 2 cases, which showed depression of the S-T segment. Among the remaining 36 patients, the $T$ wave had become inverted in 18, depression of the $S-T$ had increased in 12, while in 6 patients the added changes assumed the form of right bundle-branch block in 2, a deep S wave in leads II, III, and IIIR in 2, atrial fibrillation in 1, and a $Q$ wave in leads CR1 and CR2 in another.

When the minor changes in the premonitory electrocardiogram were least obvious, as instanced by depression of the S-T segment, confined to leads III and IIIR, cardiac pain had set in early, so that its imminence could not be predicted by the extent of the cardiographic changes.

Although the investigation has established that in every patient visited by cardiac pain from cardiac infarction the electrocardiogram can detect the presence of material coronary arterial disease years in advance, what has yet to be determined is how to tell from this premonitory tracing the time, soon or late, when cardiac pain from extension of the infarct will take place. It is likely that the exercise electrocardiogram will foretell this in the majority of cases.

Should the future discover means of halting or materially retarding the progress of coronary arterial disease, the premonitory electrocardiogram will have inestimable value. In the meantime it is held that this finding should not be disclosed to the patient nor allowed to shape his future course or career.

\section{REFERENCES}

Evans, W. (1961). Electrocardiography as a means of presaging cardiac pain. Brit. Heart. J., $23,669$. Robb, G. P., and Marks, H. H. (1964). Latent coronary artery disease. Amer. J. Cardiol., 13, 603.

Schor, S. S., Clark, T. W., Parkhurst, L. W., Baker, J. P., and Elsom, K. A. (1964). An evaluation of the periodic health examination: the findings in 350 examinees who died. Ann. intern. Med., 61, 999. 